\title{
Lusioersily
}

\section{Behavioural Usage Analysis of a Reminiscing App for People Living with Dementia and their Carers}

Mulvenna, M., Gibson, A., McCauley, C., Ryan, A., Bond, R., Laird, L., Curran, K., Bunting, B., \& Ferry, F.

(2017). Behavioural Usage Analysis of a Reminiscing App for People Living with Dementia and their Carers. In Unknown Host Publication (pp. 35-38). Association for Computing Machinery.

https://doi.org/10.1145/3121283.3121289

Link to publication record in Ulster University Research Portal

\section{Published in:}

Unknown Host Publication

Publication Status:

Published online: 22/09/2017

DOI:

10.1145/3121283.3121289

\section{Document Version}

Publisher's PDF, also known as Version of record

\section{General rights}

Copyright for the publications made accessible via Ulster University's Research Portal is retained by the author(s) and / or other copyright owners and it is a condition of accessing these publications that users recognise and abide by the legal requirements associated with these rights.

\section{Take down policy}

The Research Portal is Ulster University's institutional repository that provides access to Ulster's research outputs. Every effort has been made to ensure that content in the Research Portal does not infringe any person's rights, or applicable UK laws. If you discover content in the Research Portal that you believe breaches copyright or violates any law, please contact pure-support@ulster.ac.uk. 


\section{Behavioural Usage Analysis of a Reminiscing App for People Living with Dementia and their Carers}

\author{
Maurice Mulvenna \\ School of Computing \& Mathematics, \\ Ulster University \\ Newtownabbey BT37 0QB, UK \\ md.mulvenna@ulster.ac.uk \\ Assumpta Ryan \\ School of Nursing, Ulster University \\ Magee Campus \\ Derry/Londonderry BT48 7JL, UK \\ aa.ryan@ulster.ac.uk \\ Kevin Curran \\ School of Computing \& Intelligent \\ Systems, Ulster University \\ Derry/Londonderry BT48 7JL, UK \\ kj.curran@ulster.ac.uk
}

\author{
Aideen Gibson \\ School of Nursing, Ulster University \\ Magee Campus \\ Derry/Londonderry BT48 7JL, UK \\ a.gibson@ulster.ac.uk
}

Raymond Bond

School of Computing \& Mathematics,

Ulster University

Newtownabbey BT37 0QB, UK

rb.bond@ulster.ac.uk

\author{
Brendan Bunting \\ School of Psychology, Ulster \\ University \\ Coleraine Campus \\ Coleraine BT52 1SA, UK \\ bp.bunting@ulster.ac.uk
}

\author{
Claire McCauley \\ School of Nursing, Ulster University \\ Magee Campus \\ Derry/Londonderry BT48 7JL, UK \\ c.mccauley2@ulster.ac.uk
}

\author{
Liz Laird \\ School of Nursing, Ulster University \\ Magee Campus \\ Derry/Londonderry BT48 7JL, UK \\ ea.laird@ulster.ac.uk
}

Finola Ferry

The Bamford Centre, Ulster University

Belfast Campus

Belfast BT15 1ED, UK

f.ferry@ulster.ac.uk

\begin{abstract}
The potential for technology to support memory or enhance cognition for people living with dementia is an area of significant interest. The use of traditional reminiscence memory books and latterly digital systems to facilitate reminiscing has been shown to have some benefit for people living with dementia. However, there is a lack of research that explores how people living with dementia actually interact with digital reminiscence systems. This paper presents a new contribution to knowledge from work-inprogress in a feasibility study of facilitated reminiscence for people with dementia and results show promise in understanding the behaviour of users of interactive technologies.
\end{abstract}

\section{CCS CONCEPTS}

\footnotetext{
Permission to make digital or hard copies of all or part of this work for personal or classroom use is granted without fee provided that copies are not made or distributed for profit or commercial advantage and that copies bear this notice and the full citation on the first page. Copyrights for components of this work owned by others than ACM must be honored. Abstracting with credit is permitted. To copy otherwise, or republish, to post on servers or to redistribute to lists, requires prior specific permission and/or a fee. Request permissions from Permissions@acm.org.

ECCE 2017, September 19-22, 2017, Umeå, Sweden

(C) 2017 Association for Computing Machinery.

ACM ISBN 978-1-4503-5256-7/17/09...\$15.00

https://doi.org/10.1145/3121283.3121289
}

- Applied computing $\rightarrow$ Health care information systems - Human-centered computing $\rightarrow$ Empirical studies in HCI

\section{KEYWORDS}

Usage analysis, behavioural analysis, event log analysis, reminiscence, dementia, Alzheimer's disease, reminiscence app.

\section{ACM Reference format:}

Mulvenna, M.D., Gibson, A., McCauley, C.O., Ryan, A.A., Bond, R., Laird, E.A., Curran, K.J., Bunting, B., Ferry, F. 2017. Behavioural Usage Analysis of a Reminiscing App for People Living with Dementia and their Carers. In Proceedings of European Conference on Cognitive Ergonomics 2017 (ECCE 2017), Umeå, Sweden, September 19-22, 2017, 4 pages. DOI: $10.1145 / 3121283.3121289$.

\section{INTRODUCTION}

Dementia is a collective term for different brain disorders that are normally progressive, chronic and eventually severely impair cognitive function. The short-term memory of people living with dementia is often heavily affected, which can result in confusion and anxiety. However, often the lifetime memories of people living with dementia are still retrievable by them, especially for those at earlier stages of the condition. Reminiscence activity has been used for many years in order to support the individual to retrieve these memories and promote shared reminiscing with others [6]. This recall of past memories is an important activity in making sense of one's life [4]. This paper outlines a feasibility 
study of facilitated reminiscence for people living with dementia. The focus of this paper, however, is on a subset of that research, specifically, a study on the behavioural analysis of a reminiscing app called 'InspireD' used by people living with dementia and their carers.

\section{Facilitated Reminiscence Research}

The aim of the feasibility study was to investigate the effects of individual specific reminiscence activity facilitated through the use of the InspireD app on people living with dementia and their carers. A user centred design process philosophy [8] was used to guide the development of the app for use in the homes of the people living with dementia. Then a quasi-experimental study incorporating a paired sample of 28 dyads (person living with dementia and their carer), using repeated measures design was conducted with each participant serving as his or her own control. The research had both quantitative and qualitative components as well as a health economic analysis. The project applied several scales at start, mid- and end-point of a 12-week use of the InspireD app. Some work on the efficacy of usability scales for people living with dementia arising from the work undertaken in this project has already been published [5]. However, this paper describes another particular component of the overall project, comprising the use of event logging software built into the InspireD app, thereby enabling the measurement of use of the features of the app as well as the behaviour of the dyads involved in the study over the 12-week period of dyad use.

\section{Related Work}

The main focus of research on the analysis of event logs for computing systems used in assistive technology has been to aid in usability analysis [13] or to reveal usage patterns in web browsing behaviour [10]. Research has also been carried out to explore how rehabilitation devices can have data or event logging incorporated, but this has been more to support the goal of device monitoring [7]. The goal of incorporating event logging into the InspireD reminiscing app was to help understand behaviour and usage about how people living with dementia and their carers actually reminisce when presented with video, audio and images that may be personal artefacts or generic artefacts.

\section{The InspireD Reminiscence App}

The size, capacity and low cost of ubiquitous devices and mobile tablet computers have made them an attractive option for designing and delivering reminiscence systems. As part of a feasibility study into the benefits of individual specific reminiscence, a cross-platform device-agnostic tablet application (called 'InspireD') was developed to facilitate reminisce for people with early to moderate dementia. The two primary functions of this app are to enable people living with dementia and their carers to gather together and store selected generic and personalised memorabilia (photographs, videos, sounds, music) and to provide easy access to these visual and audio-visual cues to support individual reminiscence.

InspireD was developed with design input from the Reminiscence Network Northern Ireland and a lead user dyad (1 person living with dementia and their carer). An agile software development approach [2] was adopted to allow a functional prototype to be created early in the development lifecycle. The app incorporates local facilities for persistent data storage in SQLite database and facilitates the use of 3rd party Application Programming Interfaces (API) for Flickr and YouTube (Fig. 1).

The app consists of a user interface that is responsive across a variety of mobile devices (tablets, mobile phones). The users can upload images, video clips and audio clips to the app. The main user interface consists of a screen for people living with dementia to upload files with help, initially from an IT trainer and latterly from a family carer. A multi-screen layout allows users to choose which memorabilia they wish to access, view photos, watch videos or listen to audio files and browse selected resources.

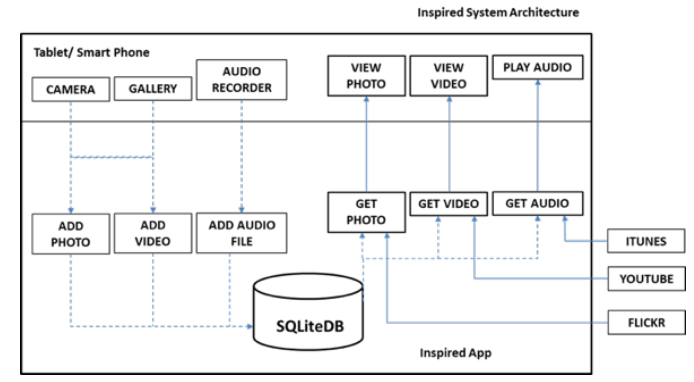

Figure 1: InspireD app system architecture

The design is minimalist, using verbal descriptors as well as images and icons to reinforce and indicate functionality to the user. Data are organised and presented primarily in the form of on-screen menus. The welcome screen is a simple login screen where the user confirms their identity by clicking a photo of themselves. The user data is contained within a local SQLite database, which can be easily queried with reporting services enabled. Multimedia reminiscing resources (photo/ video/ audio) are also stored locally in the app data directory. The app periodically sends information about usage events via email transport to a server where the event logs for all dyads were stored and collated securely.

\subsection{Event Logging Design}

People living with dementia and their careers were provided with training on the InspireD app, including the setting up of their personalised access, which was set up as a photo of themselves, which, when clicked logged them into InspireD. The InspireD app was designed to incorporate a logging facility for key events by users across 45 specific activities, covering five different types of events. The five different canonical events include: Entry (Logging in), Admin (Adding a photo, deleting an audio, etc.), Reminiscing (Viewing a video, viewing a photo, etc.), In the Moment (ITM) questions and Exit (Logging out). Thus, the 
behaviour of users can be analysed within and across each usage session, over the 12-week trial. The 'In the Moment' questions comprise items from the primary outcome measure for the study, the Mutuality Scale developed by Archbold et al. [1]. These results are beyond the scope of this short paper.

\section{RESULTS AND DISCUSSION}

User events have been classified into five main areas as shown in Fig. 2. This figure shows that the app was primarily used for reminiscing. A total of $71 \%$ of interactions from people living with dementia were within the reminiscing sections of the system whereas only $47 \%$ of interactions from carers were within the reminiscing sections $(\mathrm{p}<0.001)$. It is reassuring that people living with dementia mainly used the system for reminiscing. The data indicates that the training provided at the beginning of the home use phase where the carer was trained in being a de facto administrator for the management of reminiscing material on the app held true throughout the trial period. It is noteworthy that home usage of the app was preceded by 5 reminiscence training sessions which were provided to carers and people living with dementia by facilitators from the Reminiscence Network Northern Ireland. This usage is also reflected in the event usage data, with the intended reminiscing users of the app being primarily people living with dementia, as expected. Fewer users exit than enter to use the app but part of the training was helping users to understand how to exit the app by arbitrarily closing it ('swiping up') which resulted in no event data recorded of the exit having occurred. Power running out and other practical issues also explain this 'enter-exit' difference. Only carers could carry out 'Admin' events such as adding a photo, as mandated by their access rights set at login. It can perhaps be seen as a positive sign that carers generally chose to put into action their training and use the administrative features and add to the music, pictures and videos that were uploaded to the app prior to the intervention beginning, rather than simply browsing those already there. Fig. 3 shows that there were more interactions with photographs in comparison to music and video. Reminiscing, with its history in photograph-based memory books, has been more about the image than music, sound or video, and this effect may be what is being seen in this data [14]. What is also interesting in this data is the popularity of music to people living with dementia. Again, this is known from the literature [11,9] and anecdotally from carers of people living with dementia but it is useful to see this behaviour replicated in this trial data. Research has shown the positive effect of music on people living with dementia [3].

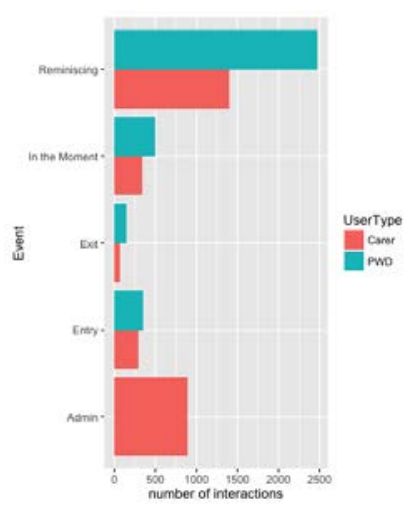

Figure 2: Number of Figure 3: Number of interactions by carers and people living with dementia (PWD) users in each of the classified events

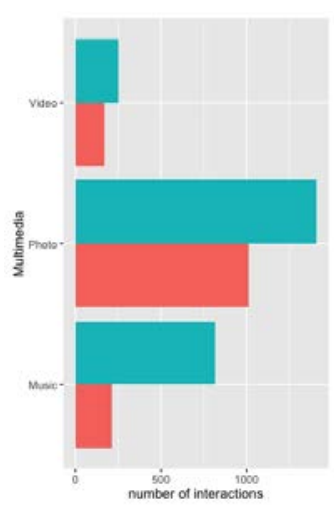

interactions by carers and people living with dementia in each of the multimedia features
The training provided at the onset of the trial provided step-bystep instructions for uploading personal reminiscing material as well as explaining how to access non-personal material via the InspireD app's access to third-party media sources including Flickr and YouTube. Therefore, both personal and generic media were accessible in the app for reminiscing.

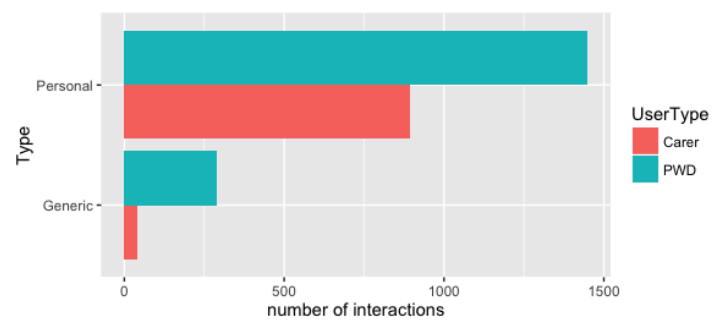

Figure 4: Number of personal and generic interactions by carers and people living with dementia

However, Fig. 4 shows that the app was primarily used for reminiscence using personal multimedia content as opposed to generic photos and videos. This is consistent with the findings of a systematic review by Subramaniam and Woods which reported that the use of general reminiscence materials was associated with no psychosocial benefit but that where the reminiscence materials and memory prompts were individual specific or involved a life review process, some immediate and longer-term psychosocial benefits related to mood, well-being and aspects of cognitive function were apparent [12].

Fig. 5 clearly shows the most popular times that the dyads of people living with dementia and carers prefer to use the app, which peaks around 11am, 3pm and 8pm. These times correspond to post-breakfast, post-lunch and post-evening meal times. 


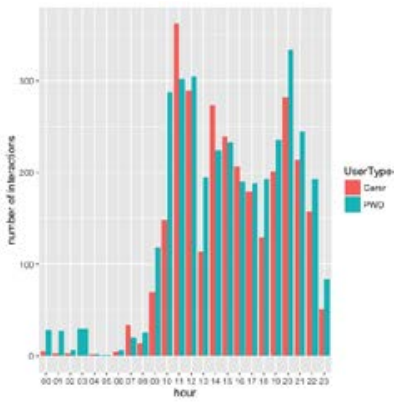

Figure 5: Number of event interactions by carers and people living with dementia per hour

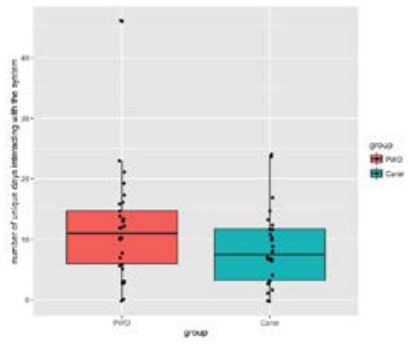

Figure 6: Number of unique day interactions by carers and people living with dementia
Fig. 5 also shows the correlation between hour of usage by both carers and people living with dementia. Moreover, we calculated the number of unique days in which users interacted with the system (refer to Fig. 6), and there is a significant statistical correlation between the number of days the carer interacted with the system and the number of days the dyad's corresponding person living with dementia interacted ( $r=5.77$, $\mathrm{p}<0.001)$. The people living with dementia showed some independence as they had slightly more unique day interactions than carers as indicated in Fig. 6. Over the 12 weeks, on average, a person living with dementia interacted with the system on $13.73 \%$ of the days which is equivalent to one reminiscence session per week. We also calculated the median frequency of exclusive day usage (or median interval between each usage day), which was 7.10 days - in other words, if a person living with dementia engaged in reminiscing on the app on a given day they are not likely to use again until one week later.

\section{CONCLUSIONS}

This paper presents the results of the analysis of event usage behavioural data of people living with dementia and their carers using a reminisce app called InspireD. The study makes a new contribution to the knowledge in the use of digital healthcare and wellbeing technologies, and shows significant promise in the use of event logging in assistive technology in order to inform the understanding of the usage of the app by users.

The analysis of the usage event log data confirms that the InspireD app was primarily used for reminiscing and that people living with dementia used it more than carers for this purpose. It also confirms that the carers also continued in the role for which they were trained, as app administrators adding personal photos, videos and music to the app. While music, videos and photos were all used to reminisce, photos were the most popular choice for people living with dementia as well as carers. Music was very popular for people living with dementia, much more so than for carers. In terms of personal versus generic media used for reminiscing, personal media was much more popular for both types of users. However, people living with dementia reminisced with personal media significantly more than carers. There is a significant statistical correlation between the number of days the carer interacted with the system and the number of days the dyad's corresponding person living with dementia interacted. The frequency of use of the app for reminiscing was about once per week over the 12- week trial period.

\section{ACKNOWLEDGMENTS}

App co-created with input from the Alzheimer's Society, Reminiscence Network Northern Ireland (RNNI) and people living with dementia and their careers. Special thanks to Alex Turnbull, Software Engineer from Kainos Software Limited for invaluable assistance in technology selection decisions. The authors gratefully acknowledge the funding support provided by HSC R\&D Grant COM/5016/14.

\section{REFERENCES}

[1] P.G. Archbold B.J. Stewart M.R. Greenlick T. Harvath. 1990. Mutuality and preparedness as predictors of caregiver role strain. Research in Nursing \& Health. 13: 375-384.

[2] M.N. Aydin, F. Harmsen, K van Slooten and R.A. Stegwee. 2005. On the Adaptation of An Agile Information Systems Development Method, Journa of Database Management Special issue on Agile Analysis, Design, and Implementation, 16(4):20-24.

[3] R.A. Bruer, E. Spitzriagel, C.R. Cloninger. 2007. The temporal limits of cognitive change from music therapy in elderly persons with dementia or dementia-like cognitive impairment: A Randomized controlled trial, J. of Music Therapy, vol. 44, pp. 308-328.

[4] H Chaudhury. 2003. Quality of life and place-therapy. Journal of Housing for the Elderly 17(1), 85-103.

[5] A Gibson, C.O. McCauley, M.D. Mulvenna, A.A. Ryan, E.A. Laird, K.J. Curran, B. Bunting, F. Ferry, R.B. Bond. 2016. Assessing Usability Testing for People with Dementia, REHAB-2016 Workshop - 4th Workshop on ICTS for Improving Patients' Rehabilitation Research Techniques, ACM Digital Library.

[6] F Gibson. 2011. Reminiscence and Life Story Work: A Practice Guide. London: Jessica Kingsley Press.

[7] K. Miller, P.J. Woollam, G. Powell, D. Hitchings, D., and J. Stallard. 2007. A Rehabilitation Device Data Logging System, Disability and Rehabilitation: Assistive Technology 2(1):9-14.

[8] Adrian F Payne, Kaj Storbacka, and Pennie Frow. 2008. "Managing the CoCreation of Value.” Article. Journal of the Academy of Marketing Science 36 (1): 83-96. doi:10.1007/s11747-007-0070-0.

[9] A. Raglio, G. Bellelli, D. Traficante, M. Gianotti, M.C. Ubezio, D. Villani, M. Trabucchi. 2008. Efficacy of music therapy in the treatment of behavioral and psychiatric symptoms of dementia, Alzheimer Disease and Associated Disorders, 22(2):158-162.

[10] Vagner Figuerêdo de Santana, M. Cecília C. Baranauskas. 2010. Summarizing observational client-side data to reveal web usage patterns. In Proceedings of the 2010 ACM Symposium on Applied Computing (SAC '10). ACM, New York, NY, USA, 1219-1223.

[11] A. Sixsmith, G. Gibson. 2007. Music and the wellbeing of people with dementia, Ageing and Society, vol. 27, pp. 127-145.

[12] P. Subramaniam and B Woods. 2012. The impact of individual reminiscence therapy for people with dementia: systematic review. Expert Review of Neurotherapeutics 12(5), 545-55.

[13] Daniel Woo, and Joji Mori. 2004. Accessibility: A Tool for Usability Evaluation. In book. Computer Human Interaction: 6th Asia Pacific Conference, APCHI 2004, Rotorua, New Zealand, June 29-July 2, 2004. Proceedings, edited by Masood Masoodian, Steve Jones, and Bill Rogers, 531-39. Berlin, Heidelberg: Springer Berlin Heidelberg.

[14] T. Wright. 2009. Drawn from Memory: Reminiscing, Narrative and the Visual Image, Proceedings of the First International Workshop on Reminiscence Systems (RSW-2009), Cambridge, UK, 5 September, 2009 pp:37-42. 